\title{
Kyasanur Forest disease virus non-mouse animal models: a pilot study
}

\author{
A. M. Nikiforuk ${ }^{1}$, K. Tierny ${ }^{2}$, T. A. Cutts ${ }^{1}$, D. K. Kobasa ${ }^{2,3}$, S. S. Theriault ${ }^{1,4}$ and B. W. M. Cook ${ }^{1,5^{*}}$
}

\begin{abstract}
Objectives: Mouse models have delivered variable recapitulation of Kyasanur Forest disease (KFD) pathology and consistently demonstrated neurological involvement which may be a limited feature of human disease. With the purpose of more accurately modelling human disease progression we infected several small-mammalian models: guinea pigs, hamsters and ferrets with a titered infectious dose of Kyasanur Forest disease virus (KFDV). Clinical indicators of disease severity were observed for seventeen days, on day eighteen a visual post-mortem analysis of visceral organs was conducted. Viral load in selected tissues was measured to infer disease signs and the establishment of viral replication.

Data description: Daily monitoring did not reveal any observable signs of illness; weight loss was minimal across species and gross pathology did not indicate severe viral infection. Tissue specific tropism and establishment of viral infection was monitored by quantitative real-time polymerase chain reaction (qRT-PCR). No viral replication was detected in ferrets $(n=0 / 3)$, but was present in the spleen of guinea pigs $(n=3 / 3)$ and the brain of hamsters $(n=3 / 3)$. Low levels of viral RNA were detected in multiple hamster tissues (kidney, liver, lung and spleen) suggesting the possibility of viral tropism and possible adaptation to the host. No serological tests were performed.
\end{abstract}

Keywords: Kyasanur Forest disease virus, Tick-borne, Animal model, Flavivirus, Guinea pig, Hamster, Ferret

\section{Objective}

In humans, Kyasanur Forest disease (KFD) is described as a biphasic illness that presents with flu-like symptoms in the first stage and may advance to a second stage with hemorrhagic fever manifestations and/or neurological abnormalities [1, 2]. Epidemiological studies and case reports have not provided clear evidence to ascertain if neurological symptoms are a common or rare sequalae in recovered individuals [3-6]. Attempts to establish viral replication in a mouse model have shown the absence of fever and progression of neurologic disturbance with viral RNA [7], viral antigen [8] and infectious virus [9] being detected in the brain. The high prevalence of neurological infection in mice may contradict human symptoms

\footnotetext{
*Correspondence: bcook@cytophage.com

${ }^{5}$ Present Address: Cytophage Technologies Inc, 26 Henlow Bay, Winnipeg, MB R3Y 1G4, Canada

Full list of author information is available at the end of the article
}

of disease progression. Thus, potentially making such models poor indicators of pathology, viral tropism and of diminished use in screening prophylaxis or vaccine candidates. This pilot study investigates the establishment of viral infection and etiologic pathology in non-mouse models (guinea pig (Cavia porcellus), hamster (Mesocricetus auratus) and ferrets (Mustela putorius furo)). Animals challenged with an infectious dose of Kyasanur Forest disease virus (KFDV) were monitored daily for visual signs of disease, febrility (ferret only), lethality, and weight loss. Post-mortem examination was performed to collect tissue from the: brain, kidney, liver, lung, spleen of each animal. Viral RNA was extracted from tissue samples and quantified by real-time RT-PCR. Presence of viral RNA was interpreted as evidence of viral tropism and replication. We hypothesized that one of the selected non-mouse models may act as a better model for human disease of KFDV infection than current mouse models. 
Table 1 Overview of data files/data sets

\begin{tabular}{llll}
\hline Label & Name of data file/data set & File types (file extension) & Data repository and identifier (DOI or accession number) \\
\hline Data File 1 [12] & KFD_CT.csv & .CSV & figshare (https://doi.org/10.6084/m9.figshare.12115797) \\
Data File 2 [13] & KFD_GE.CSV & .CSV & figshare (https://doi.org/10.6084/m9.figshare.12115803) \\
Data File 3 [14] & KFD_KM.csV & .CSV & figshare (https://doi.org/10.6084/m9.figshare.12115812) \\
Data File 4 [15] & KFD_R_Rmd & .Rmd & figshare (https://doi.org/10.6084/m9.figshare.12115818) \\
\hline
\end{tabular}

\section{Data description}

\section{Virus preparation and animal infection}

KFDV isolate P9605 [10] was previously passaged nine times in suckling mouse brains, and two times within the Containment Level 4 (CL-4) Laboratory (Canadian Science Centre for Human and Animal Health, Winnipeg Canada) in Vero E6 cells. Viral supernatant was harvested from the second Canadian passage to obtain stocks for challenge of the non-mouse models.

Each animal species was housed separately in groups of three per cage: female (28-70 days old) outbred LVG Golden Syrian hamsters (Charles River Laboratories, New York, USA), female (26-58 weeks old) outbred Hartley guinea pigs (Charles River Laboratories, New York, USA) and female (650-800 g) specific pathogenfree (SPF) ferrets (Marshall BioResources, New York, USA) with subcutaneous implanted IPTT-300 temperature and ID transponder devices (BioMedic Data Systems Inc., USA). The procedures described herein, were approved by the Canadian Science Centre for Human and Animal Health-Animal Care Committee (animal use document H-14-010) adhering to the Canadian Council on Animal Care guidelines. Animals were fed and monitored daily for a 7-day acclimation period and for the duration of the experiment. Pre-challenge, each animal was anesthetized with $5 \%$ isoflurane in oxygen and $0.1 \mathrm{~mL}$ with $6.3 \times 10^{4} \mathrm{TCID}_{50}$ units of passagetwo KFDV was intra-peritoneal-injected. The challenge dose was back titrated on Vero E6 cells. Observations of visual disease, weight loss and lethality were conducted for 16 days (hamsters and guinea pigs) or 17 days (ferrets). For ferrets, temperature was also recorded via the subcutaneous IPTT-300 sensors.

At the end of the observation period, animals were anesthetized with $5 \%$ isoflurane in oxygen and blood was collected. While under anesthesia, animals were euthanized (intra-cardiac injection of $120 \mathrm{mg} / \mathrm{kg}$ of pentobarbital) for gross pathology and tissue collection. Isolated tissues (brain, kidney, liver, lung and spleen) were inactivated with RLT and viral RNA was extracted with the RNeasy Plus Mini kit (Qiagen, Mississauga, Canada). Viral RNA was detected via quantitative real-time polymerase chain reaction (qRT-PCR) using the Roche LightCycler ${ }^{\circledR} 480$ RNA Master Hydrolysis Probes and a previously published primer design [11, 12].

\section{Kyasanur Forest disease animal modelling}

Throughout the course of observation, clinical signs of disease (ruffled fur, hunched posture, lethargy or loss of appetite) were not observed and all animals gained weight [14]. Temperature measurements from the ferrets fluctuated between 37.7 and $40.3{ }^{\circ} \mathrm{C}$ [14]. Upon post-mortem analysis, there were no apparent lesions or significant differences in organ weight. Assessment of viral RNA in isolated tissue samples (brain, kidney, liver, lung and spleen) revealed an absence of infection in ferrets (below limit of detection) and could only be found in the spleens of guinea pigs $(\mathrm{Ct}=30.33-30.87)$ [13]. In contrast, viral RNA was detected across all sampled hamster tissues, with the exception of a single kidney sample. Viral RNA was detected in hamster brain tissue $(\mathrm{Ct}=31.7-32.7)$ but not that of guinea pigs or ferrets $(\mathrm{Ct}=>40)$ [13] (Table 1). All data analysis was done in $\mathrm{R}$ version 3.6.3 [15].

\section{Limitations}

This pilot study contains several limitations including: small sample size, no replication for statistical inference, single dose-inoculation, and the presence of infectious virus cannot be directly inferred from the detection of nucleic acid [16]. Additionally, the P9605 isolate that was disseminated from India to many CL-4 laboratories in North America may be mouse-adapted [9] and is not a low passage isolate. From these findings we suggest that future attempts to model human-like disease from KFDV infection should consider: hamsters or immuno-compromised hamsters, a low passage wild-type isolate of KFDV or one rescued from an infectious clone system, should be inoculated for dose response, supernatants from tissue extraction should be passaged in a naïve animal to select for viral adaptation to the host. This pilot study demonstrates that hamsters could be preferred over guinea pigs or ferrets as a potential small non-mouse model of KFD pathology. It is unknown whether infected hamsters will 
recapitulate single phase or biphasic disease course and pathology.

\begin{abstract}
Abbreviations
KFD: Kyasanur Forest disease; KFDV: Kyasanur Forest disease virus; P9605: Passaged human isolate of Kyasanur Forest disease virus; $\mathrm{CL}-4$ : Containment level 4; SPF: Specific pathogen-free; RNA: Ribonucleic acid; qRT-PCR: Quantitative real-time polymerase chain reaction; IPTT: Implantable programmable temperature transponder; $\mathrm{TCID}_{50}$ : Median tissue culture infectious dose assay.
\end{abstract}

\section{Acknowledgements}

We would like to thank the Special Pathogens Program, Public Health Agency of Canada for access to the containment level 4 laboratory and program support, specifically Lisa Fernando, Kaylie Tran and Anders Leung). We would also like to thank Cytophage Technologies Inc for payment of the article processing charge.

\section{Authors' contributions}

BWMC and TAC prepared and titrated virus inoculum and performed qRT-PCR. KT and DKK performed the inoculation and autopsies. All authors performed animal husbandry, routine work in CL-4 and assisted in preparing the manuscript. AMN helped prepare the manuscript and assembled the data. BWMC, DKK and SST received ethical approval for the study and conceived the design. All authors read and approved the final manuscript.

\section{Funding}

Funding was provided to Dr. Steven S. Theriault as an operational budget of the Public Health Agency of Canada. The funding body had no input in the study design, analysis, data interpretation, writing or in the decision to publish the manuscript.

\section{Availability of data materials}

The data described in this Data note can be freely and openly accessed on figshare (https://figshare.com/) under:

https://doi.org/10.6084/m9.figshare.12115797 [12], https://doi.org/10.6084/ m9.figshare.12115803 [13], https://doi.org/10.6084/m9.figshare.12115812 [14], https://doi.org/10.6084/m9.figshare.12115818 [15].

\section{Ethics approval and consent to participate}

Ethical approval was granted by the Canadian Science Centre for Human and Animal Health Animal Care Committee (\#H-14-010).

\section{Competing interests}

The authors have no competing interests or conflicts of interest.

\section{Author details}

${ }^{1}$ Applied Biosafety Research Program, National Microbiology Laboratory, Public Health Agency of Canada, 1015 Arlington Street, Winnipeg, MB R3E 3R2, Canada. ${ }^{2}$ Special Pathogens Program, National Microbiology Laboratory, Public Health Agency of Canada, 1015 Arlington Street, Winnipeg, MB R3E 3R2, Canada. ${ }^{3}$ Department of Medical Microbiology, and Infectious Diseases, The University of Manitoba, 745 Bannatyne Avenue, Winnipeg, MB R3E 0J9, Canada. ${ }^{4}$ Department of Microbiology, The University of Manitoba, 213 Buller Building, Winnipeg, MB R3T 2N2, Canada. ${ }^{5}$ Present Address: Cytophage Technologies Inc, 26 Henlow Bay, Winnipeg, MB R3Y 1G4, Canada.

\section{References}

1. Munivenkatappa A, Sahay R, Yadav P, Viswanathan R, Mourya D. Clinical and epidemiological significance of Kyasanur forest disease. Indian J Med Res. 2018;148(2):145. http://www.ijmr.org.in/text. asp?2018/148/2/145/243689.

2. Holbrook MR. Kyasanur forest disease. Antivir Res. 2012;96(3):353-62. https://doi.org/10.1016/j.antiviral.2012.10.005.

3. Work TH, Trapido H, Murthy DPN, Rao RL, Bhatt PN, Kulkarni KG. Kyasanur forest disease. III. A preliminary report on the nature of the infection and clinical manifestations in human beings. Indian J Med Sci. 1957;11(8):619-45.

4. Webb HS, Rao RL. Kyasanur forest disease: a general clinical study in which some cases with neurological complications were observed. Trans R Soc Trop Med Hyg. 1961;55:284-98.

5. Adhikari Prabha MR, Prabhu MG, Raghuveer CV, Bai M, Mala MA. Clinical study of 100 cases of Kyasanur Forest disease with clinicopathological correlation. Indian J Med Sci. 1993;47(5):124-30.

6. Sadanandane C, Elango A, Marja N, Sasidharan PV, Raju KHK, Jambulingam P. An outbreak of Kyasanur forest disease in the Wayanad and Malappuram districts of Kerala, India. Ticks Tick Borne Dis. 2017;8(1):2530. https://doi.org/10.1016/j.ttbdis.2016.09.010.

7. Dodd KA, Bird BH, Jones MEB, Nichol ST, Spiropoulou CF. Kyasanur Forest disease virus infection in mice is associated with higher morbidity and mortality than infection with the closely related Alkhurma hemorrhagic fever virus. PLoS ONE. 2014;9(6):e100301.

8. Basu A, Yadav P, Prasad S, Badole S, Patil D, Kohlapure RM, et al. An early passage human isolate of Kyasanur Forest disease virus shows acute neuropathology in experimentally infected CD-1 mice. Vector-Borne Zoonotic Dis. 2016;16(7):496-8.

9. Sawatsky B, McAuley AJ, Holbrook MR, Bente DA. Comparative pathogenesis of Alkhumra hemorrhagic fever and Kyasanur Forest disease viruses in a mouse model. PLoS Negl Trop Dis. 2014;8(6):e2934.

10. Cook BWM, Cutts TA, Court DA, Theriault S. The generation of a reverse genetics system for Kyasanur Forest disease virus and the ability to antagonize the induction of the antiviral state in vitro. Virus Res. 2012;163:431-8.

11. Mourya DT, Yadav PD, Mehla R, Barde PV, Yergolkar PN, Kumar SRP, et al. Diagnosis of Kyasanur forest disease by nested RT-PCR, real-time RT-PCR and IgM capture ELISA. J Virol Methods. 2012;186(1-2):49-54. https://doi. org/10.1016/j.jviromet.2012.07.019.

12. Nikiforuk, Aidan. KFD_CT.csv. figshare https://doi.org/10.6084/m9.figsh are.12115797. v1 (2020)

13. Nikiforuk, Aidan. KFD_GE.csv. figshare https://doi.org/10.6084/m9.figsh are.12115803. v1 (2020).

14. Nikiforuk, Aidan. KFD_KM.csv. figshare https://doi.org/10.6084/m9.figsh are.12115812. v1 (2020)

15. Nikiforuk, Aidan. KFD_R.Rmd figshare https://doi.org/10.6084/m9.figsh are.12115818. v1 (2020)

16. Biava M, Caglioti C, Bordi L, Castilletti C, Colavita F, Quartu S, et al. Detection of viral RNA in tissues following plasma clearance from an Ebola virus infected patient. PLoS Pathog. 2017;13(1):e1006065. https://doi. org/10.1371/journal.ppat.1006065.

\section{Publisher's Note}

Springer Nature remains neutral with regard to jurisdictional claims in published maps and institutional affiliations. 